\title{
Feasibility of optical waveguide immunosensors for pesticide detection: physical aspects
}

\author{
E.F. Schipper, R.P.H. Kooyman, R.G. Heideman, J. Greve \\ MESA Research Institute, Department of Applied Physics, Bio-Interface Group, University of Twente, PO Box 217, 7500 AE Enschede, \\ Netherlands
}

\begin{abstract}
The feasibility of detecting small molecules such as pesticides using optical evanescent-wave sensors is discussed with emphasis on the Mach-Zehnder sensor and a newly developed sensor called a 'critical' sensor. For direct detection of an estimated average pesticide layer growth of $2 \times 10^{-4} \mathrm{~nm}$, the sensitivity of the Mach-Zehnder sensor is almost adequate $\left(1 \times 10^{-3} \mathrm{~nm}\right.$ within one hour), whereas that of the 'critical' sensor $\left(2 \times 10^{-2} \mathrm{~nm}\right)$ is not sufficient. However, the simplicity of this latter sensor is very attractive. Results of $\alpha$-hSA/hSA immunoreaction experiments obtained with this last type of sensor are presented.
\end{abstract}

Keywords: Immunosensors; Optical waveguides; Pesticides

\section{Introduction}

The frequent employment of pesticides for achieving high agricultural yields may result in the contamination of drinking water, as traces of pesticide residues enter the soil. Pesticides accumulate in animal tissue and can cause diseases at high concentration. The maximum concentration for a single kind of pesticide in drinking water permitted by the $\mathrm{EC}$ guidelines is $100 \mathrm{ng}^{-1}$ (European Drinking Water Act of 1980). This small concentration can only be detected with very sensitive but laborious and time-consuming methods (such as HPLC, gas chromatography, etc.). For this reason, the development of new, small, cheap and fast sensor systems is desirable. Here, evanescent-wave optical sensing techniques could offer a promising solution. These sensors are based on measuring a variation of the refractive index within the evanescent-field volume of a surface plasmon or waveguide mode supported by some metal or dielectric. Typically the penetration depth is a few hundreds of nanometres from the sensor surface. As has been demonstrated previously [1-3], antibodies can be immobilized on the surface of such a device, providing an optical immunosensor with sensitivities of the order of $\mathrm{pg} \mathrm{mm}^{-2}$ for the analyte of interest.

Antibodies capable of specific recognition of certain classes of pesticides have been developed and their use in competitive evanescent-wave sensors has been demonstrated [4,5]. Here we assess the possibility of developing an evanescent-wave sensor based on direct detection of these small molecules.

\section{Pesticide detection}

Pesticides are small molecules with molecular weight (MWs) ranging from 0.2 to $0.5 \mathrm{kDa}$. The immunoreaction between the pesticides and the tailored antibodies, immobilized on the waveguide surface, results in an average layer growth directly related to the size of the analyte molecules. Using a rough theoretical estimate related to the experimentally determined monolayer thickness [6], we calculated that an intact antibody $\left(\mathrm{MW} \approx 150 \mathrm{kDa}\right.$ ) covers approximatcly $36 \mathrm{~nm}^{2}$. Then the resulting maximum available density for pesticide binding sites $(\alpha)$ is $2 \times 0.027 \mathrm{~nm}^{-2}$ (one antibody has two binding sites). For a pesticide concentration $[P]$ of $100 \mathrm{ng}^{-1}\left(2 \times 10^{-10} \mathrm{M}\right)$, and an affinity constant $(K)$ of $10^{8} \mathrm{M}^{-1}$, the fraction $(I)$ of bound pesticide is $\approx K[P]$. Assuming that the size of a molecule is directly related to its $\mathrm{MW}$, we model a pesticide molecule as a cube with a volume $(V)$ of $0.216 \mathrm{~nm}^{3}$. The immunoreaction will then give a maximum average layer growth

$t_{\mathrm{f}}^{\prime}=\alpha V[P] K$ 
In this way a layer growth of $2 \times 10^{-4} \mathrm{~nm}$ is found. This is comparable to an analyte coverage of $0.12 \mathrm{pg}$ $\mathrm{mm}^{-2}$. The sensitivity of surface plasmon resonance (SPR) sensors is not high enough to measure this layer growth [1]. The non-lossy character of waveguide modes opens possibilities for more sensitive sensors [7]. The grating coupler sensor [2] and resonant mirror sensor [3] are examples, but do not take optimal advantage of the non-lossy character of the propagating modes, so that the resulting sensitivities are not much better than that obtained with an SPR sensor. In the following the use of two other types of waveguide sensors will be discussed.

\section{Potential sensor configurations}

\subsection{The Mach-Zehnder interferometer sensor}

The operating principle of a Mach-Zehnder interferometer sensor has been described before [6-8]. The light is split and coupled in reference and sensor channels with equal intensities. If in the sensor channel the analyte molecules can bind to the surface, the effective refractive-index contrast in this channel will change, thereby changing the intensity of the interference signal after outcoupling and combining. The intensity of the static interference pattern at the position of the detector is given by [6]

$I=I_{0}\left[1+\cos \left(\frac{2 \pi}{\lambda} \Delta N_{\mathrm{eff}} L\right)\right]$

Here, $L$ is the interaction length, $\lambda$ is the vacuum wavelength, and $\Delta N_{\text {eff }}$ is the effective refractive-index difference experienced by the two beams.

As can be deduced from Eq. (2), an attractive aspect of this sensor is that the sensitivity increases with long interaction lengths. A more detailed description of this sensor is presented by Lechuga et al. [6], With $L=1$ $\mathrm{cm}$ the phase drift of this system is $0.01 \times 2 \pi$ per hour. This drift was found to be the main factor determining the overall sensitivity. An average layer growth of $1 \times 10^{-3} \mathrm{~nm}$, corresponding to an analyte coverage of $0.6 \mathrm{pg} \mathrm{mm}{ }^{-2}$, could be detected. For direct pesticide detection the sensitivity of this sensor is almost enough. However, a disadvantage of this system is the complexity in adjustment prior to a measurement, and signal interpretation due to the periodicity of the signal. Therefore, we have investigated whether a new simpler sensor, based on analyte-induced beam deflection, can provide an alternative.

\subsection{The 'critical' sensor}

The basic concept of the 'critical' scnsor is depicted in Fig. 1. Part of the waveguide layer outside the triangle

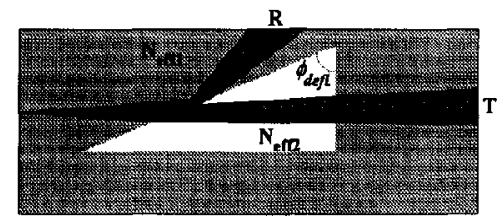

Fig. 1. The 'critical' sensor.

is covered with cladding, so that in this area the evanescent field built up by the waveguide mode is shielded from an immunoreaction. A guided mode strikes the interface between the shielded and unshielded areas, resulting in a deflection of the beam. When the mode strikes the interface with an incident angle above the so-called critical angle, the light is totally reflected. By virtue of Snell's law this critical angle is a function of the effective refractive-index contrast. This index contrast and, thereby, the critical angle, will change as the result of an immunoreaction.

A divergent beam is coupled into the waveguide. This beam arrives at the interface, when in a balanced situation half of the light is reflected and half of it is transmitted. A change in the critical angle, as a result of a change in the effective refractive-index contrast, shift this intensity balance:

$I=T-R=\int 2 \frac{\partial T}{\partial \theta_{\mathrm{c}}} \frac{\partial \theta_{\mathrm{c}}}{\partial N_{\mathrm{efP} 2}} \partial N_{\mathrm{eff} 2}$

Here $\theta_{c}$ is the critical angle and $N_{\text {cffz }}$ is the effective refractive index in the unshielded area. Presently, an average layer growth of $2 \times 10^{-2} \mathrm{~nm}\left(12 \mathrm{pg} \mathrm{mm}^{-2}\right)$ can be measured with this system. More details will be published elsewhere [9].

\section{Materials and methods}

The planar waveguides were fabricated using standard techniques ( $\mathrm{Si} / \mathrm{SiO}_{2} / \mathrm{Si}_{3} \mathrm{~N}_{4} / \mathrm{SiO}_{2}$ ). The high refractive index of the waveguide layer (LPCVD $\mathrm{Si}_{3} \mathrm{~N}_{4}, n=2.00$ ), in contrast to those of the aqueous cladding ( $n=1.33$ ) and substrate layer (thermally oxidized $\mathrm{SiO}_{2}, n=1.46$ ), gives at a waveguide layer thickness of $170 \mathrm{~nm}$ a $\mathrm{TM}_{0}$ spatial electric-field distribution with a good confinement near the waveguide layer surface [7]. After fabrication, the waveguides were partly covered with a cladding layer ( $\mathrm{PECVD}, \mathrm{SiO}_{2}, n=1.46$ ). Analyte layer growth then only influences the waveguiding properties in the uncovered area of the waveguides.

An overview of the critical sensor set-up can be seen in Fig. 2. A $2.5 \mathrm{~mW}$ chopped HeNe laser beam is endfire coupled to the device using an $f=10 \mathrm{~mm}$ cylindrical lens. At the sensor interface the light splits into a reflected $(R)$ and a transmitted $(T)$ part. The intensity balance between $(R)$ and $(T)$ is tuned with a rotatable 


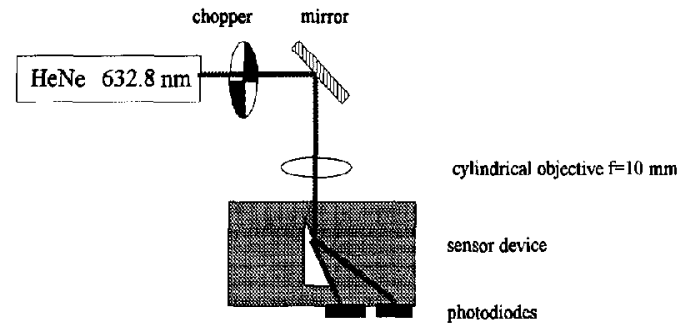

Fig. 2. The 'critical' sensor configuration.
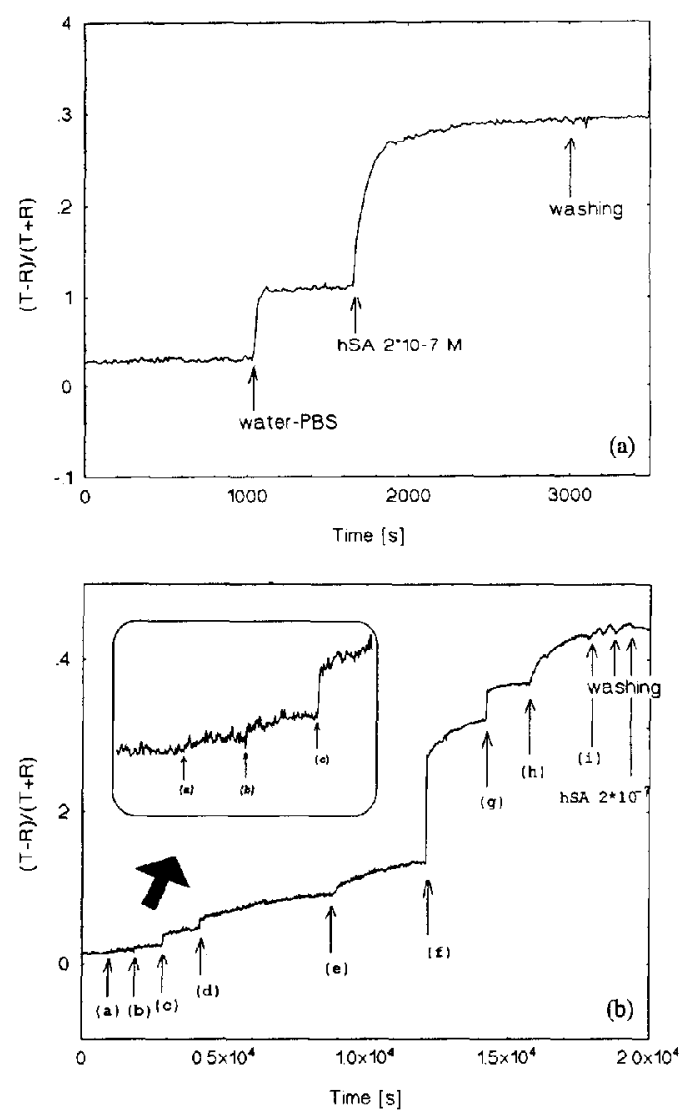

Fig. 3.(a) Preparation of the waveguide surface, water-PBS sensitivity check and immobilizing of the hSA receptor layer on the waveguide surface. (b) Immunoreaction experiments. Different concentrations of $\alpha$-hSA in PBS solution are added: (a) $1 \times 10^{-9} \mathrm{M}$, (b) $4 \times 10^{-9}$ $\mathrm{M}$, (c) $1 \times 10^{-8} \mathrm{M}$, (d) $2 \times 10^{-8} \mathrm{M}$, (e) $4 \times 10^{-8} \mathrm{M}$, (f) $1.5 \times 10^{-3}$ M, (g) $2.5 \times 10^{-7} \mathrm{M}$, (h) $4.7 \times 10^{-7} \mathrm{M}$, (i) $8 \times 10^{-7} \mathrm{M}$.

mirror. The intensities of both the beams are measured with a photodiode. The pre-amplified signals are added or subtracted, and amplified with a lock-in amplifier, before being further processed with a computer. The $(T-R)$ signal is the variable signal. The $(T+R)$ signal is used as a normalization for this $(T-R)$ signal.
To test the 'critical' sensor, immunoreaction experiments were performed with the well-known $\alpha$-hSA/ hSA system (Sigma). The waveguide surface was coated with a dichlorodimethylsilane layer, resulting in a hydrophobic surface. Subsequently an hSA layer was immobilized by physical adsorption from a PBS solution, with a concentration of $2 \times 10^{-7} \mathrm{M}$ hSA, until the adsorbed layer reached an average thickness $\approx 2 \pm 1$ $\mathrm{nm}$. In the immunoexperiments different concentrations of $\alpha$-hSA were added to the cuvette.

\section{Results and discussion}

In Fig. 3 a representative example of an immunoreaction is shown. First an hSA layer is adsorbed (Fig. $3(a)$ ). With an assumed refractive index of 1.45 for the hSA, the adsorbed protein layer thickness could be estimated by comparing the changing signal $(T-R)$ with that when the waveguide was immersed in glucose solutions of known refractive index [9]. Subsequently, different concentrations of $\alpha$-hSA were added to the cuvette solution. The resulting time response of the system is depicted in Fig. 3(b), (for details see the caption of Fig. 3(b)). It should be noted that due to the manual adding of different concentrations the kinetics are not well controlled, and consequently only the steady-state values can be interpreted. The antibody layer grown after a full immunoreaction, resulting in a bound fraction $\Gamma=1$, is $5 \pm 1 \mathrm{~nm}$. This layer thickness is consistent with a monolayer coverage of the $\alpha$-hSA molecules. In Fig. $4 \Gamma$ as a function of the $\alpha$-hSA concentration is depicted. From this an affinity constant of $1.4 \times 10^{-7} \mathrm{M}^{-1}$ can be estimated. These experiments demonstrate the feasibility of the critical sensor for the detection of immunoreactions. However, the obtained detection limit of $2 \times 10^{-2} \mathrm{~nm}$ is not sufficient for direct detection of nanomolar quantities of small-molecularweight molecules.

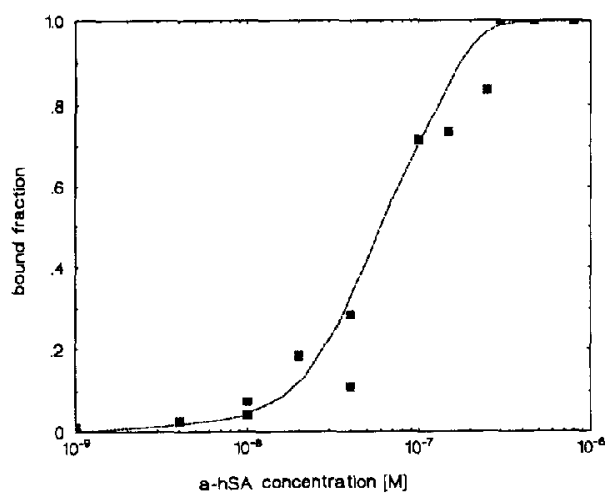

Fig. 4. Bound fraction vs. $\alpha$-hSA concentration. The affinity constant of the $\alpha-h S A / h S A$ system is evaluated as $1.4 \times 10^{7} \mathrm{M}^{-1}$. 


\section{Conclusions}

The Mach-Zehnder immunosensor has a proven detection limit of $1 \times 10^{3} \mathrm{~nm}$ [6]. The 'critical' sensor has a detection limit of $2 \times 10^{-2} \mathrm{~nm}$. Although the strong points of the 'critical' sensor are its simplicity of adjustment and signal interpretation, it does not seem adequate for the detection of low concentrations of small molecules. However, in view of its very simple design it could be an attractive candidate for a competitive immunoassay. The Mach-Zehnder sensor shows the highest sensitivity, and is, therefore, at this moment more suitable for direct pesticide detection. An increase of its sensitivity should be possible with a reduction and integration of the optical parts of the sensor. Also a chemical modification of the sensor surface, for example, by immobilization of the proteins inside a hydrogel matrix as used in the SPR Biacore system [10], will appreciably increase the responsitivity.

\section{Acknowledgement}

These investigations have been supported by the Netherlands Technology Foundation (STW).

\section{References}

[1] R.P.H. Kooyman, A.T.M. Lenferink, R.G. Eenink and J. Greve, Vibrating mirror surface plasmon resonance immunosensor, Anal. Chem., 63 (1991) 83.

[2] D. Clerc and W. Lukosz, Sensitivity of integrated optical grating and prism couplers as (bio)chemical sensors, Sensors and $A C$ tuators $B, 11$ (1993) 273-284.

[3] R. Cush, J.M. Cronin, W.J. Stewart, C.H. Maule, J. Molloy and N.J. Goddard, The resonant mirror: a novel optical biosensor for direct sensing of biomolecular interactions. Part 1: Principle of operation and associated instrumentation, Biosensors Bioelectron., 8 (1993) 347-353.

[4] P. Krämer and R. Schmid, Flow injection immunoanalysis (FIIA) - a new immunoassay format for the determination of pesticides in water, Biosensors Bioelectron., 6 (1991) 239-243.

[5] F.F. Bier and R.D. Schmid, Real time analysis of competitive binding using grating coupler immunosensors for pesticide detection, Biosensors Dioelectron., 9 (1994) 125-130.

[6] L.M. Lechuga, A.T.M. Lenferink, R.P.H. Kooyman and J. Greve, Feasibility of cvanescent wave interferometer immunosensors for pesticide detcction: chemical aspects, Sensors and Actuators B 24-25 (1995) 762-765.

[7] W. Lukosz, Principles and sensitivities of integrated optical and surface plasmon sensors for direct affinity sensing and immunosensing, Biosensors Bioelectron., 6 (1991) 215-225.

[8] R.G. Heideman, R.P.H. Kooyman and J. Greve, Performance of a highly sensitive optical waveguide Mach-Zehnder interferometer immunosensor, Sensors and Actuators B, 10 (1993) 209-217.

[9] E.F. Schipper, R.P.H. Kooyman and J. Greve, to be published.

[10] S. Löfås, M. Malmqvist, I. Rönnberg, E. Stenberg, B. Liedberg and I. Lundströn, Bioanalysis with surface plasmon resonance, Sensors and Actuators B, 5 (1991) 79. 